\title{
Aided Nonverbal Communication through Physical Expressive Objects
}

\author{
Stephanie Valencia \\ HCI Institute, Carnegie Mellon \\ University \\ svalenci@andrew.cmu.edu \\ Cynthia L. Bennett \\ HCI Institute, Carnegie Mellon \\ University \\ cbennet2@andrew.cmu.edu
}

\author{
Mark Steidl \\ Disability Rights Advocate, Disability \\ Rights Pennsylvania \\ mark.steidl@verizon.net \\ Jeffrey P. Bigham \\ HCI Institute, Carnegie Mellon \\ University \\ jbigham@cmu.edu
}

\author{
Michael L. Rivera \\ HCI Institute, Carnegie Mellon \\ University \\ mlrivera@andrew.cmu.edu \\ Henny Admoni \\ Robotics Institute, Carnegie Mellon \\ University \\ henny@cmu.edu
}

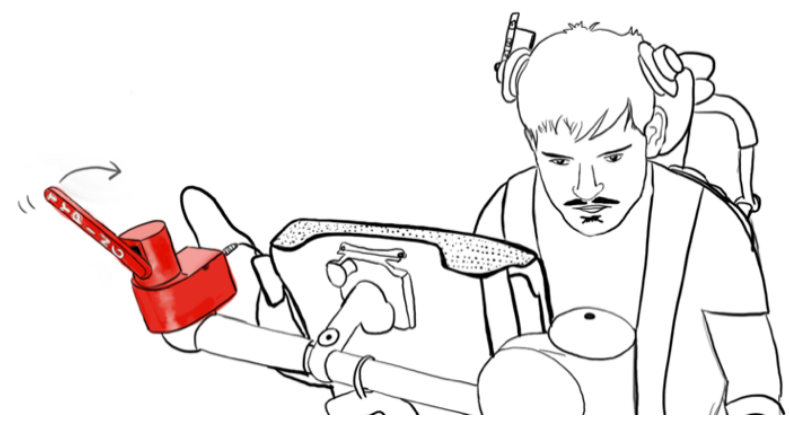

Figure 1: A design team including an augmented communicator, his close conversation partners and HCI researchers worked together on designing a physical expressive object to support AAC-based communication.

\begin{abstract}
Augmentative and alternative communication (AAC) devices enable speech-based communication, but generating speech is not the only resource needed to have a successful conversation. Being able to signal one wishes to take a turn by raising a hand or providing some other cue is critical in securing a turn to speak. Experienced conversation partners know how to recognize the nonverbal communication an augmented communicator (AC) displays, but these same nonverbal gestures can be hard to interpret by people who meet an AC for the first time. Prior work has identified motion-based $\mathrm{AAC}$ as a viable and underexplored modality for increasing ACs' agency in conversation. We build on this prior work to dig deeper into a particular case study on motion-based AAC by co-designing a physical expressive object to support ACs during conversations. We found that our physical expressive object could support communication with unfamiliar partners. As such, we present our process and resulting lessons on the designed object itself and the co-design process.
\end{abstract}

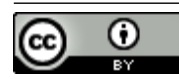

This work is licensed under a Creative Commons Attribution International 4.0 License.

ASSETS '21, October 18-22, 2021, Virtual Event, USA

(c) 2021 Copyright held by the owner/author(s).

ACM ISBN 978-1-4503-8306-6/21/10.

https://doi.org/10.1145/3441852.3471228

\section{CCS CONCEPTS}

- Human-centered computing; • Accessibility technologies; • Empirical studies in interaction design.;

\section{KEYWORDS}

Access technologies, Communication, Disability, Tangible interfaces

\section{ACM Reference Format:}

Stephanie Valencia, Mark Steidl, Michael L. Rivera, Cynthia L. Bennett, Jeffrey P. Bigham, and Henny Admoni. 2021. Aided Nonverbal Communication through Physical Expressive Objects. In The 23rd International ACM SIGACCESS Conference on Computers and Accessibility (ASSETS '21), October 18-22, 2021, Virtual Event, USA. ACM, New York, NY, USA, 11 pages. https://doi.org/10.1145/3441852.3471228

\section{INTRODUCTION}

Augmentative and alternative communication (AAC) encompasses all types of communication we use to express ourselves in addition to speech. AAC includes facial expressions, sign language, gestures, written language, and even Morse code [2]. Aided forms of AAC use technology to support alternative communication modes, such as picture or letter boards that people can point to or speech generating devices people can use to compose messages $[4,10]$. Commercial speech generating AAC systems are currently only customizable at the word selection and speech production levels, and they do 
not yet support augmentations that can increase nonverbal communication. Nonverbal communication is key in helping regulate turn-taking, convey personality, and execute actions that increase social agency [24], all which are current challenges for communication device users. For instance, an augmented communicator (AC) using an AAC device, is compelled to respond within the synchronous timing constraints of in-person interactions even though they use an asynchronous text-based medium [18]. ACs have to compose a message on their device using text and then they share their message with text-to-speech while a non-augmented conversation partner responds synchronously using speech without needing to compose a message. This creates an interaction asymmetry $[17,35]$. The type of relationship with a communication partner (e.g., years knowing each other) can also impact an AC's communication and participation [43]. Knowledgeable communication partners know they should wait and allow time for an AC's response, and that they can support ACs by repairing communication breakdowns between an AC and a non-augmented speaker [43]. In contrast, people meeting an $\mathrm{AC}$ for the first time might not wait long enough and may not be aware of an AC's nonverbal language that can be key in achieving mutual understanding [12, 13, 25].

Prior work in human-computer interaction (HCI) and humanrobot interaction (HRI) indicates that physical devices can achieve communicative effects with motion. For example, robotic objects using motion can communicate nonverbal cues [27], and even influence the sentiment of an interaction [1,29]. Physical devices can be designed such that motion is used to draw attention only when needed and otherwise exist in the periphery of conversation [28], which foreground robots as suitable to assist with conversations between people, for instance, during conflict $[19,22]$, or when mediating conversation between autistic children and their conversation partners [33]. Prior work has also identified motion-based communication modalities as viable and underexplored to support conversations between ACs and non-augmented conversation partners. Specifically, physical expressive objects were proposed as a form of motion-based AAC that can increase an AC's agency by helping ACs convey a precise message, enable timely participation, and bring attention to an augmented communicator when needed [42]. Despite the advantages that motion provides in nonverbal communication, it remains a relatively unexplored output communication modality for AAC systems.

In this work we collaborated with an augmented communicator, our co-author Mark, and his close communication partners to design a fully functional physically expressive object to support his specific conversational goals. We carried out an in-depth case-study style design where HCI researchers worked closely over several months to customize a prototype to meet Mark's needs. We then evaluated remotely how the device was used at home for two months with a three-week diary study data collection. We present the following contributions: (1) an in-depth case study on designing a new type of AAC with an AC collaborator, (2) the design of a motion AAC device, and (3) an evaluation of our design in context. Furthermore, we learned important aspects to consider when carrying out remote co-design including the need to establish what won't work accesswise upfront, stay practical to avoid ideas that will be unusable, and devising a long-term bespoke device maintenance plan post-design.

\section{BACKGROUND AND RELATED WORK}

Our work is situated at the intersection of augmentative and alternative communication research, human-robot interaction, and participatory design.

\subsection{Nonverbal Communication in AAC}

Augmented communicators use a variety of nonverbal gestures to communicate with partners but often, only skilled conversation partners are able to pick up on these cues [25]. Close conversation partners to an $\mathrm{AC}$ who know their augmented partner well can support and facilitate ACs communication with others by translating nonverbal gestures to unfamiliar partners and helping resolve misunderstandings [5, 43]. Some partners even use nonverbal behaviors as a strategy to help ACs participate in conversations with others. For instance, some partners move their body to orient themselves towards the AC to encourage others to interact with the AC directly [43]. In this work, we leverage the versatile language of motion to explore the design of a device that can use nonverbal expression to support AC's communication when interacting with others.

Nonverbal gestures produced by ACs have been used in prior work as access methods to control a device. Trained machine learning models that understand facial expressions and enable gestures to be shortcuts for communication have been used to convert AC's gestures into actions through computer vision and machine learning approaches $[14,26]$. We recognize that ACs already have and use nonverbal communication everyday but in our work, we explore how nonverbally expressive objects can support AC's agency in conversation when talking to partners with different levels of familiarity with ACs.

Finally, prior work has explored the concept of using an external robotic object as a form of aided motion-based AAC by examining what conversational aspects these devices could help with, what they could look like, and how they could move to support an AC's communication. Through a co-design workshop, researchers, puppeteers, ACs, and their caregivers explored potential uses, forms, and behaviors for socially assistive sidekicks for AAC [42]. Assistive Sidekicks were proposed as a secondary animated agent that could support an AC achieve a specific goal, for example by moving in a way that could show others the AC wanted to participate at a given time in a conversation. We built on this work to carry out an extended exploration with one augmented communicator to design a sidekick that will support his specific conversational goals.

\subsection{Expressive Objects for Communication}

Moving physical objects can support communication by conveying emotions that can be interpreted as social cues. Physical expressive objects or "machines" have been designed to enhance remote communication in times of social distancing [39]. Tangible signals made of folded paper shapes that track data and display it through embodied motion have been used as peripheral information displays [15]. Additionally, robotic objects used at the periphery of the conversation, accompanying a conversation instead of being direct participants of it, have shown to influence a conversation's dynamics and impact human behavior. For example, MicBot, a peripheral robotic object resembling a microphone, demonstrated that 
balancing participation and improving group performance could be achieved without playing an explicit participatory role in a group. MicBot sat on a table and followed the current speaker with its body and nonverbally encouraged participation by orienting and tilting towards the person that had been speaking the least [40]. Similarly, Kip1, an object-like robot tracked the aggressiveness of a conversation and conveyed an emotional response with the aim of evoking empathy and behavior change in couples having an argument [19]. Kip1 resembled a lamp and was designed to accompany the conversations in a way that did not distract from it, motivating our work in exploring expressive robotic objects as companions that can influence conversations and enhance human interaction without distracting or replacing the main interaction among human partners.

\subsection{Augmenting AAC-based Interaction}

To address communication challenges between an augmented communicator and a non-augmented partner, prior work has focused on finding ways to make communication more efficient by adding context awareness to AAC devices [23] and enabling close partners to help ACs complete a message using a companion app [9]. Other advances have centered around increasing partner awareness. For example, companies and researchers have developed partners screens [41] and awareness and status displays [9, 38] that enable speaking partners to view when an AC is composing a message, and to view other feedback in the form of emojis and lights. In addition to technology, human partners such as aides are often needed to assist the AAC user and conversation partner. Human aides can augment the interaction between an $\mathrm{AC}$ and a speaking partner by reminding another speaker about the AC's intent or elaborating on answers on behalf of the AC [43]. The trade-off for the AAC user when having a human aide supporting their conversation is a loss of independence to shape communication as desired [9, 21, 43]. This work motivated us to explore how to increase partner awareness and opportunities for ACs to gain more agency in conversations through a motion-based AAC sidekick.

\section{APPROACH AND METHOD}

In this work we engage in participatory design [34] to co-design a bespoke technology with an augmented communicator and his close conversation partners. We carried out research through design, in which we engaged in designing as a research activity to obtain design knowledge in the process [45], positioning design activities as data collection and analysis opportunities [30]. Our activities included information gathering through interviews, surveys, prototyping sessions, and diary entries over a period of 12 months. We documented our decision-making process that occurred over several in-person and remote sessions. We captured every conversation, meeting, and activity with notes, daily reflections, artifacts, or video and audio recordings when permitted among all involved co-designers. We collected all online exchanges including pictures, emails, and discussions we had on remote collaboration tools. All audio recordings were transcribed and artifacts were thematically analyzed.

\subsection{Design Team Participants}

This work involved a design team including Mark, an expert augmented communicator who is also a co-author of this work, Mark's close conversation partners, and university HCI researchers. We describe our participants:

Mark, augmented communicator. Mark has used AAC for over 19 years and has long advocated for AAC users by working at a world leader AAC device company, serving as a student government senator, and advocating in a variety of disability rights campaigns. Mark has cerebral palsy and is a wheelchair user. $\mathrm{He}$ uses indirect selection to control his AAC device. That is, Mark's AAC device scans through each option on the device (e.g., word, letter, shortcut) until Mark presses his head switch to select a target. In addition to his device, Mark uses facial expressions and eye blinks to communicate.

T and D, Mark's family members. T and D facilitated our remote design meetings, sharing about their experiences communicating with Mark, and facilitating the exchange of materials needed to carry out this work.

University HCI researchers. HCI researchers with backgrounds in accessibility, fabrication, and robotics coordinated the design activities.

\section{DESIGN PROCESS}

This section describes our design process, detailing the design decisions made and highlighting design values we needed to consider when designing a motion-based form of AAC. We describe: (1) our discovery of design opportunities for nonverbal expressive sidekicks, (2) the definition stage during which we set out to discuss design priorities for a future functional sidekick, (3) the development of different ideas and prototypes, (4) a delivery stage in which we pilot tested the device in context and refined our prototype, and (5) the evaluation stage during which we tested the prototype for two months, with three weeks of diary study collection.

\subsection{Discovery: Opportunities for Expressive Objects in AAC}

We invited Mark to co-design a physical expressive sidekick for his use. Mark had been a participant in a past study and had expressed interest in exploring a solution that would allow him to better capture his communication partner's attention when needed and without interrupting the ongoing conversation. Mark also noted that he would mainly want to use his sidekick during group conversation, in a classroom setting or student government meeting. Some challenges he experiences with group conversations involve turn-taking such as knowing when to interrupt a group or being able to tell others he is working on a message before conversation partners assume he has nothing else to contribute and move on to the next topic. The time it takes Mark to compose a message can vary between a couple of seconds to up to minutes and therefore he often asks for more time to compose a message to remind partners to wait. 
At the time we started this work, Mark was completing his last semester of college and was attending student government meetings for which he acted as vice-president. He invited us to observe one of his meetings so we could get a better sense of how his current group conversations developed. Unfortunately, the COVID-19 pandemic commenced during this time and the student government meetings were canceled. Mark shared via email a little more about the inperson meetings:

"The conversation is fast paced, and sometimes it's difficult for me to contribute. However, the other senators are tuned into how my communication system works. As vice-president, I'm responsible for keeping time so I have the following preprogrammed phrases: 'I'm sorry to interrupt, but we're running out of time; Let's get back on track. We can always discuss this at a later time if necessary.' 'I would like to add something to the conversation. It will take me a few minutes to write it. Would you call on me in a few minutes?"'

Upon further discussion with Mark and his family, we learned that he uses a variety of strategies to let others know he wants to compose a message and then later, that he is ready to share it. For instance, Mark had shared written guidelines with his professors on how to facilitate a discussion in a class where someone uses a communication device with suggestions including giving Mark some questions ahead of time so that he can prepare an answer, or allowing Mark to give a one-word answer that the professor can build on. Another solution Mark and his family came up with was to build a switch-activated LED light strip mounted right next to Mark's wheelchair's head rest. The LED light strip was made by D. T explained that Mark turns on his light to indicate he is ready to participate: "He turns it on to alert the professors he has an answer, [the light] is currently acting as his own sidekick when he is in class." The light was a nonverbal way to call for attention and indicate to others that Mark was ready but, it did not allow for more nuanced communication. We decided to explore how we could signal other social cues with motion to support Mark in managing turn-taking in group conversations.

\subsection{Definition: Goals and Sidekick Properties}

The design team met to discuss specific properties the sidekick should have to best support Mark's interactions and conversations with different partners. In this section we report on the identified goals and possible useful scenarios in which Mark envisioned using the sidekick.

To scaffold our definition phase, the HCI researchers selected specific properties to discuss covering the function, social factors, and aesthetics surrounding the sidekick: (1) what conversational goals should the sidekick support? (2) How would the sidekick be controlled? (3) How should the sidekick be introduced in the conversation? (4) What should the sidekick do while it is inactive? and (5) What should the sidekick look like? We used these discussion points as probes to imagine different possibilities and identify design constraints.

4.2.1 Defining accessible sidekick controls. We learned that the preferred mode to control a potential sidekick would need to involve Mark's head-switch. The HCI researchers had brainstormed a series of controllers to discuss with Mark ranging from manual inputs to automatic sensing mechanisms, but when discussing these ideas, we learned that he had already tried and discarded many of these input modes before. T explained that Mark has mixed muscle tone due to his athetoid cerebral palsy, so other input modes that require motor precision and repetition of controlled movements such as eye tracking, facial gestures or foot pedals are not accessible.

"We have tried a lot of access points; can I go over them? we tried the elbow; we tried the knee. We tried some things with [Mark]'s hands. . he can go in one direction but can't retreat from that direction so if he were to get his hand out here it might stay there and then but really, he needs to [bring it back] to release it as a switch. [Mark]'s most functional area for selecting is his head."

We also learned that gesture-based input could be tiring. Mark and his family had also tried a system that comprised of a wearable headband with electrodes that could sense winks and specific facial grimaces that could be detected and help Mark with accessing his communication device. They were trying to use it as an alternative to eye gaze but it was impossible to find a consistent facial movement. Having to do repetitive gestures was also physically taxing. Mark currently has two head-switches, one to control his AAC device and another one to turn his light on and off. Mark stated this was already the maximum number of switches he desired so he would prefer to be able to use the same switch he uses for his light, for the sidekick. So, we integrated the sidekick to be controllable by one of his head switches.

4.2.2 Sidekick interactions and inactive state. It was challenging to talk about sidekick properties and interaction without having a physical model of what the sidekick could look like. Mark expressed that the word "prototype" the HCI researchers kept using to describe a possible sidekick was not completely clear to him so $\mathrm{HCI}$ researchers clarified they meant a model of what the sidekick could be. Clarifying that nothing was set in stone yet allowed us to freely explore the possibilities and talk about the constraints of each. We discussed how we imagined a possible sidekick would behave starting from what it would do when it is not in use and how partners would discover it. Prior to our meeting, Mark had answered that similar to his head light, the sidekick should always be present but not always active to prevent Mark from having to retrieve something that needed to be taken off and put on. We also had imagined that the sidekick could be hidden and it should appear suddenly when needed but this also begged the question as to how we could execute this mechanically. Mark indicated he would prefer for the sidekick to remain it its position when not in use. Mark shared that he would not know where to store a sidekick so he assumed it would work best for him if left on, while remaining ambient. We pinned this in our discussion and revisited it later asynchronously once we converged on a sidekick form factor.

4.2.3 Physical appearance and placement. Mark mentioned that he wanted the sidekick to have a smile, but he was unsure about what he wanted it to look like. We conducted a literature review on expressive robotic objects that had smiles or faces and used the images gathered plus other expressive objects. Some of the images we used are shown in Figure 2. We shared these different expressive 


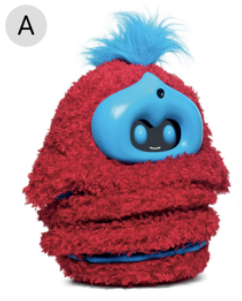

Character-like
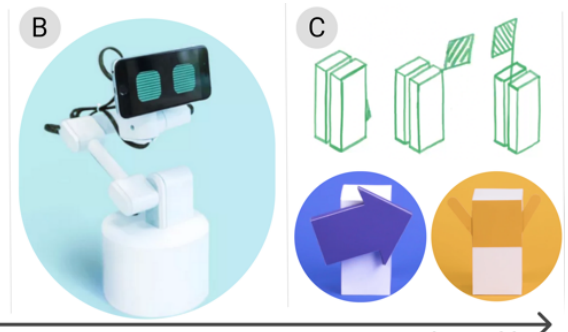

Object-like
Figure 2: Expressive robotic objects ranging from characterlike to object-like. (A) Tega, a robot emotive companion [44]image $\odot$ Bruce Peterson, (B) Emoto, an emotive AI sidekick made of a robotic stand that acts as a body for your phone [6], and (C) Paper Signals, image @Google, animated objects that track and display data through embodied motion [15].

robotic objects examples using Padlet, a digital online collaboration board maker that Mark recommended for its accessibility. We placed each image in a way that Mark could comment below it asynchronously and use thumbs up/thumbs down to prioritize the favorite ideas over ones that did not resonate with what he had in mind. We also allowed a space in the collaborative board for sketching, and for labelling where the sidekick could potentially be placed on Mark's wheelchair. While Mark did not sketch, he indicated preference for the flag-looking object from the Paper Signals project [15] that showed a flag raising up from a box-like container (Figure 2, right). Mark also indicated that it would be best to place the sidekick to one side, close to the AAC device, instead of other alternate options that included using the space in the back of Mark's head-rest. Once we decided on the sidekick's form the HCI researchers started prototyping, as described in the next section.

\subsection{Development: Ideating and Prototyping}

Once we decided on a flag-like form factor, we started exploring different possible motions. The HCI researchers created a low-fidelity prototype using a popsicle stick, paper, a servo motor, and an $\mathrm{Ar}$ duino to showcase a variety of motions and share them with Mark and his family in a video. Figure 3 shows some example motions. The motions included: (1) rise and hold (to call for attention like raising a hand); (2) home position (to demonstrate the idle state); (3) rise and wave (coming up and moving forward showing enthusiasm or agreement, like nodding yes, or calling for attention); (4) therethere motion (moving from 90 to 180 degrees slowly like saying "calm down," or "It's OK"); and (5) the metronome motion (moving from 0 to 180 degrees and then back while Mark is composing a message to show something is in process).

The HCI researchers also modeled a 3D flag-like object to ground discussions of what could be further developed in a high-fidelity prototype (Figure 4, A). We shared the video with the motions and the 3D model prototype with Mark and his family, and met to discuss the device's development further. The HCI researchers focused on learning the sidekick's size limitations and placement constraints, according to Mark's needs. To do this, we carried out a conversational, spatial brainstorm in which we relied on shared visual information to ground our discussion. The HCI researchers shared the 3D model they had created and Mark and his family pointed to different places on his chair, while rotating their camera (used during video conferencing) around to facilitate our understanding of the space available and a potential mounting place.

4.3.1 Constraining the design space. Understanding technical limitations and Mark's access preferences was key in facilitating our discussion and making decisions about the sidekick. As we brainstormed different possibilities, $\mathrm{D}$ and $\mathrm{T}$ reminded the $\mathrm{HCI}$ researchers about Mark's AAC device features and limitations. For example, Mark's AAC device has Bluetooth and infrared remotecontrol capabilities that can be used to interface with a computer or another device. Nonetheless, the infrared and Bluetooth are not always reliable. T shared that the infrared control worked better than Bluetooth but requires a specific receiver that Mark only uses when he is working at a dedicated desk. It was also important to have a conversation on how Mark prefers to use his AAC device: "Some people do use the computer side of [the AAC device]; Mark does not. He does not like shifting over to it because having the open computer where you have the language software on one side and the computer software on the other side, it slows down the language side. That is just one reason." This conversation reaffirmed our decision to use the head switch as the way to more practically control the sidekick without needing to worry about wireless connectivity issues. These led us to a discussion about how we could enable triggering specific separate motions if we only had one head switch as our input. Our conversation turned into understanding the technical limitations of our envisioned sidekick. Could we built it to move only while the button is pressed? Should it stop once Mark released the button? Should it be voice activated too? Mark expressed he did not want to complicate things too much, by saying no to different ideas about using voice to activate different motions, and having the sidekick rotate to show different colors that could mean something. Such features would add additional learning and work to Mark's daily processes by requiring him to remember numerous sidekick states and controls.

4.3.2 Motion as a new material to explore. D has experience tinkering around Mark's wheelchair. For example, he built the LED light. However, D had not considered using motors before and was surprised to find how dramatic and descriptive even small motions could be. D explained how Mark can use his light to say yes and no, two light flashes for yes, one for no, but with the motor the object could move in a specific way that means something else, even when being triggered by the same switch. "When you showed the one you showed in the video, it was quite dramatic. It was almost like waving. So, he is like waving toward himself to get somebody's attention like saying "I am ready now"." When watching the example motion in the video we discussed how the different motions could be seen differently from different angles. For example, the metronome motion is more understandable from the side than from a front view. We decided to add a second degree of freedom that could support adjusting the sidekick to be visible at different viewing angles.

We thought that the desire to have a flag-like object could mean Mark might have been interested in adding a message to it. However, during our conversational brainstorm we realized Mark and 


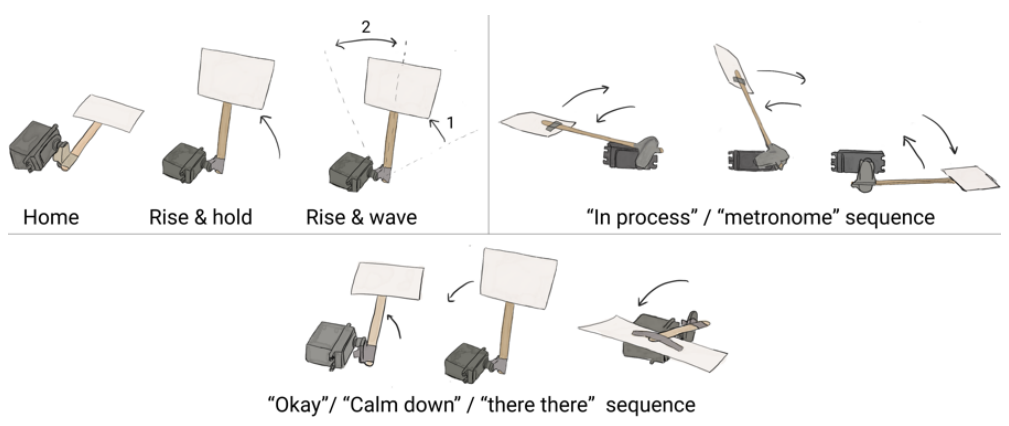

Figure 3: Low-fidelity prototype example motions.

his family considered that motions themselves were enough. D suggested extending the part attached to the motor a couple of inches higher, and removing the rectangular flag face area as it would probably be more practical, less vulnerable to wind, and make the sidekick smaller in size. Mark agreed and shared how he would like to change the square-like looking attachment to something like a popsicle stick and having the possibility of adding some extension to it in the future. D agreed: "If the part that attached to the motor, that stick, is just extended a couple of inches higher, that is almost enough of a visual cue, if it moves up and down. I don't even know if it needs a rectangular area at all."

4.3.3 Making use of the space available and contrasting colors. We also learned how having a very large sidekick could interfere with Mark's transfers out and in of the wheelchair. It was important to stay within Mark's chair perimeter to avoid obstacles. Mark had an existing mount for his wheelchair that could be used to place the sidekick right to one side of his AAC device without blocking his face or his line of sight. T and D offered to drop off the mount so that we could fabricate the sidekick around it. We decided that a sidekick with an approximate total height of 6 inches would be more than enough to be seen. Mark's family also suggested using a contrasting color to Mark's wheelchair and his accessories which are mostly all black. We decided then the sidekick would need to be a color that could stand out as D suggested: "I saw the video of the thing moving. That is going to catch people's attention. You could have just the stick and people would see that. A bright-colored stick would do the job."

\subsection{Prototype Implementation}

We implemented the ideas and feedback from our conversations into a fully functional sidekick prototype. We designed a mechanical structure made of 3D printed parts which is actuated by two micro servo motors with a range of motion up to 180 degrees of freedom each (Figure 4, C). The arm has holes that fit M3 screws and allow for easy mounting of other desired extensions. The device is connected to a metal piece that enables mounting on the wheelchair. The electrical design uses a METRO Mini 328 microcontroller from Adafruit industries and a custom PCB that connects the adaptive button and power lines to the servo motors and to the microcontroller. The device has a micro-USB connection which brings power through an external battery pack or directly from the AAC device's USB port. A mono audio jack adaptor on the device serves as the connector for
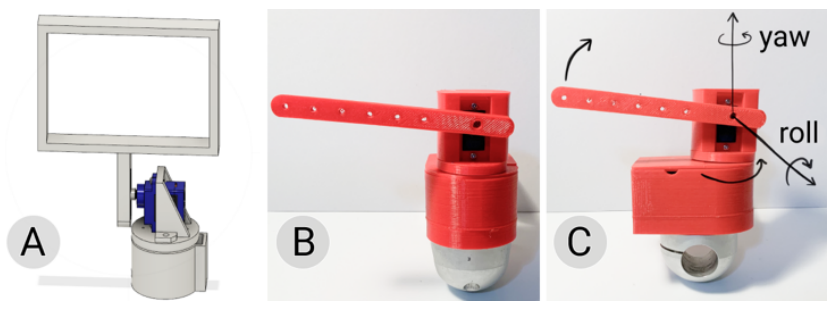

Figure 4: A) Early prototype 3D model; B) Front view and C) side view of fabricated sidekick attached to wheelchair mount.

the adaptive button. The sidekick's gestures were authored using the Arduino Servo library that allows setting motors to specific positions. To support others in creating their own sidekicks, we have open-sourced the 3D designs, software, and electronic schematics here: https://github.com/Svsquared/AAC-sidekick.

4.4.1 Initial programmed motions. Following video exchanges via email we programmed 3 motions for Mark to test including Mark's preferred motion: (1) rise, pause and wave to call for attention; and two additional motions to explore and probe for ideas: (2) a return to home motion rotating the sidekick slowly inwards, and (3) pointing outwards as if pointing to an object nearby. The sidekick also included an intro motion sequence to signal being on and receiving power: once plugged in the sidekick would turn on and move to center itself. Each motion was programmed to be activated according to different button press durations: one fast button press triggered the rise up and wave motion, a press lasting about 3 seconds moved the sidekick back to home position, and with a longer press lasting about 6 seconds the sidekick rotated to point outwards. Each of these motions began with a "preamble" sequence which would bring the sidekick's arm to the front and center of the device. This preamble was intended to capture people's attention before the sidekick carried out the main motion.

\subsection{Delivery: Testing and Iterating}

We delivered our 3D printed design for a "test run" in which Mark would try the device for a couple of weeks and provide feedback. After trying out the prototype, Mark and his family came up with a new motion they wanted to use, called timer. Mark often says "can you hold on a minute please" to indicate he would like to say 


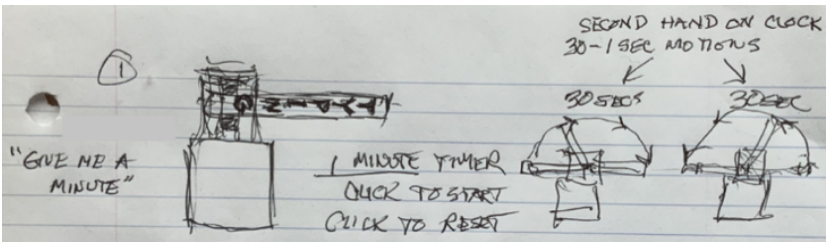

Figure 5: Hand sketch produced by $D$ explaining the new timer motion.
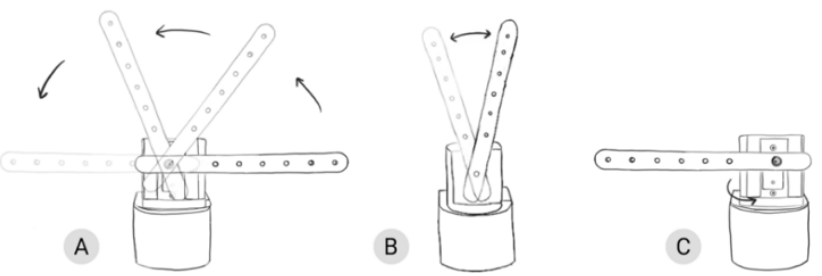

Figure 6: (A) The timer motion moves 30 positions right to left and then 30 positions left to right, advancing 6 degrees per step; (B) The wave motion waves back and forth 6 times; and $(\mathrm{C})$ the reset sequence centers the base and lowers the arm.

something and needs a minute to compose his message. The idea of the timer motion is to make the sidekick's arm act as a timer that moves from side to side at a pace of 6 degrees per second, that is 30 seconds moving from left to right and 30 seconds right to left for a total of one minute side to side (Figure 5 and 6). Another suggestion was to add the word "typing" on the sidekick's arm to further clarify the message.

Mark also found that the "preamble" sequence before each motion was more confusing than helpful in capturing people's attention so we removed the preamble entirely. We also decided to drop the pointing motion as it was rarely used. After some iterations and feedback through virtual meetings we finalized a version of the sidekick that Mark would use for a longer period of time. The final version had only two main motions-the timer motion lasting 1.5 minutes total and the wave motion. The timer and the wave could be activated by a fast click and a 3 second press respectively. Clicking the head button again during any of the motions stopped and reset the sidekick immediately (Figure 6), a key function we identified during the testing phases as it could help stop the sidekick in case of an accidental press of the head button or to stop the prolonged timer motion.

\section{EVALUATION: USE AND IMPACT}

Evaluating the sidekick for two months enabled us to understand if and how it supported Mark's communication in different realworld contexts and with different communication partners. Using the sidekick for a long period of time also provided us with ideas for improvements and allowed us to reflect on how the day-to-day tasks (i.e., technology set ups) and different communication channels (virtual or in-person) impacted the sidekick's effectiveness and use. We analyzed the diary entries provided by Mark, we graphed Mark's circle of communication partners, and we met regularly to discuss how the sidekick was used and how it worked according to different interactions. We were interested in understanding if other people understood the sidekick's purpose, if the sidekick enabled Mark to participate more in conversations, support him with turntaking and show others his intent to contribute.

We collected 11 diary entries over three weeks. We asked Mark for information on any activities during which he used the sidekick and we asked him to rate its usability and social factors including: "The sidekick worked as expected"; "it was easy to use"; "It helped me achieve what I wanted"; "it was more distracting to me than it should have been"; "partners noticed the sidekick"; "partners understood what I was conveying when using the sidekick"; and "partners seemed distracted by the sidekick". To better understand Mark's relationship with the communication partners he interacted with during the evaluation period, we interviewed Mark and created a circle of communication partners (Figure 7). His partners included family and friends. His three family members, T, D, and $\mathrm{P}$, as well as his friends, acquaintances, service providers, and the new communities he is building through his advocacy work. The partners reported in the diary study are lightly bolded in Figure 7.

The sidekick was more useful during face-to-face conversation as compared to using it in virtual meetings. We also found close communication partners did not need the sidekick but understood its purpose right away. We also learned that there were some unexpected barriers to the sidekick use like remembering to make sure it was plugged in to power and to the head switch. We present our main evaluation findings next.

\subsection{Sidekick Use and Performance}

The sidekick was reportedly used 7 of 11 days, summarized in Table 1. The sidekick was used between 2 to 5 times per conversation on 6 days and it was used between 6 to 10 times on one day when Mark was talking to his friends from an AAC conversation group. We learned that it was easy to forget to plug the sidekick in and this was the main reason for days of nonuse. The sidekick was already mounted on the wheelchair everyday but it needed to have the head switch cable connected to it to be operable, as well as making sure it was connected to the AAC device for power. One improvement suggested by $\mathrm{T}$ was to make the sidekick integrated with the device, in a way that it could be controlled with the AAC device and it could not need external cables.

Mark reported that 7 out of 7 times others noticed the sidekick during his meetings. The sidekick was easy to use and worked as expected most of the time, although for one meeting with his job coach, the sidekick worked sporadically - requiring Mark to press the head switch twice or for a little longer to make sure it started the motion. Even though the HCI researchers could not replicate the latency problem we reflected on the importance of making sure the sidekick was sensitive enough to Mark's press frequency and style, as it was evident from his report that the microcontrollers internal delays were not allowing the button to be sensitive enough to his desired rhythm of use on some occasions.

Mark reported that the sidekick helped him manage turn-taking when talking to his AAC group friends and when talking to a group of his current and past aides with whom he is close. The sidekick did not really help with turn-taking that much for his 
Table 1: List of meetings reported using the diary entry method over a period of three weeks.

\begin{tabular}{llll}
\hline ID & \# of partners & Relationship & Medium \\
\hline A & 2 & Past assistants (PA) & In-person \\
B & 5 & New and past assistants (CA, PA) & Online \\
C & 5 & New and past assistants CA, PA) & Online \\
D & 15 & Music group friends (WL) & Online \\
E & 5 & AAC group friends (AC) & Online \\
F & 1 & Job coach (JC) & Online \\
G & $15+$ & Disability rights advocates (DA) & Online \\
\hline
\end{tabular}

other conversations. The sidekick did not really increase Mark's participation in meetings but it was helpful in reminding partners to wait for Mark's response and was used frequently to replace his preprogrammed message: "can you hold on a minute please?" In general, having access to a motion-based AAC sidekick enabled a short-cut in communication, allowing others to understand typing is happening and allowing Mark to not have to verbally say "one moment please" with his AAC device.

\subsection{Close and New Communication Partners}

The familiarity with AAC and also with Mark's communication style is what makes close communication partners skilled enough to not need the help of the sidekick, nonetheless they understood its purpose right away. During a drive-in visit to some of Mark's past aides who have known him for more than 4 years and have become friends (meeting A, Table 1), Mark shared they asked what the sidekick was and he demonstrated how it worked and they "got it right away." "If they know you well, they do not need the extra help. It is sort of like a novelty but not absolutely necessary because those folks are waiting; they are patient and they are waiting. If they see Mark's body language that he is writing they know Mark is typing." Nonetheless the sidekick seemed useful when Mark talked to the same group of past aides' friends and a group of current aides (meeting B and C, Table 1) due perhaps to the fact that this meeting was online and it had more people in it, which could make turn-taking a little more challenging.

The sidekick was also useful when Mark talked with AAC friends who are familiar with the workflow involved in being an augmented speaker. Mark shared that some of them had told him they wanted one for themselves. The sidekick also seemed useful in one occasion with Mark's mother at home. Mark was in another room and he triggered the sidekick to call his mom, she heard the sound and caught it moving and realized Mark was calling for her attention.

We hypothesized that the sidekick would be more useful with unfamiliar partners, allowing Mark to better regulate turn taking dynamics with people who had not met him before but we learned that this was hard to measure in an online setting as unfamiliar partners needed to first be introduced to AAC and Mark's communication style online, which brings new constraints, in addition to having to interpret the sidekick.

\subsection{Better in-person}

The sidekick was originally designed to support face-to-face conversation but due to the COVID-19 pandemic, the group conversations

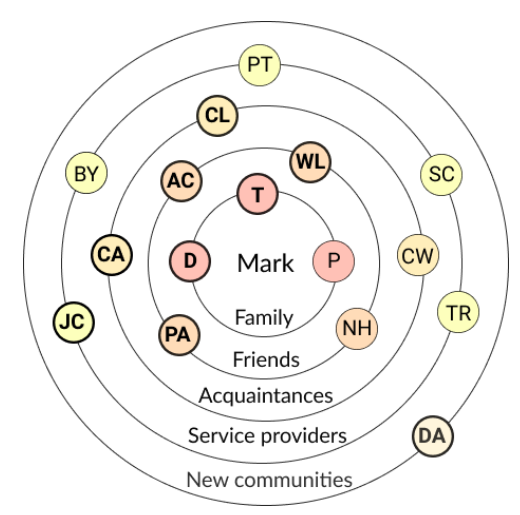

Figure 7: Parents $(D, T)$ and brother $(P)$ are included in the family circle. Friends include past aides (PA), AAC group friends (AC), music camp friends (WL) and neighbors (NH). Acquaintances include co-workers (CW), HCI collaborators (CL), and current aides (CA). Service providers include a job coach (JC), physical therapist (PT), Supports Coordinator (SC), and an assistant's agency (BY). In the outer-most circle a disability rights community (DA) has recently started to get to know Mark.

that Mark had during our evaluation period were mostly online with a couple of exceptions for when he did some drive-in visits to friend's houses or used it at home. Mark shared that the sidekick helps more with turn-taking in person than it does online. The visuals a person can get during a video call can be limited by the number of people on the call, the positioning of one's camera and people's attention to the screen. This was the case when Mark was talking to the disability rights advocates. Mark has found that the best alternative when meeting with large groups on video is to use the chat instead of the sidekick; T elaborated on his answer:

"Like today he was on a call and there were like 30 people on the call. So, he is using sidekick, your picture is small and sometimes there is not a single facilitator but the speaker is shifting around. and it's not like there is not anyone in place who is going to call on you. And in that situation, you may be better off using the chat. I know he had to do it today. He said, 'I have a question.' And said it in the chat. The visual for the zoom call in those situations where there is a lot of people and they are not $100 \%$ used to AAC, they are not going to have a reaction to the sidekick or even know what it is." 
Mark also explained that people not seeing the sidekick was also due to his camera's position. The family has worked on positioning the camera to make sure Mark's face is shown without cutting off the sidekick but it is sometimes challenging to get a good set up for every meeting. "I think it is fantastic in real-time with real people. You know, face to face. There is no question. They are seeing it. It's here, you know, but in the digital meetings, it is more difficult."

Surprisingly we did find that using the sidekick online was useful with acquaintance or "mid-circle" partners who were familiar with Mark but who were not as skilled as those partners in the first two closest circles. For example, when talking to the design collaborators on this paper on Zoom, Mark effectively used the sidekick to help others pace themselves and their questions. The sidekick also supported Mark to talk with his group of friends and current aides, and when talking to his augmented communicator friends during his AAC group by helping Mark show others he was composing a message.

\section{DISCUSSION}

As a case study, this project demonstrates how motion can support AAC interactions and conversations. We argue that motion-based AAC achieved through expressive objects is a promising new communication modality to continue exploring. Our work revealed specific ways in which a physical expressive sidekick supported an augmented communicator's interactions, uncovering additional opportunities for future work. We also learned lessons on how to scaffold co-design activities to collaborate as a team and envision and develop a new technology.

\subsection{Benefits of aided nonverbal communication}

People familiar with AAC understood the sidekick's purpose right away. Experienced communication partners did not need the sidekick to communicate with Mark as they know how to read Mark's body language and they know to patiently wait. Close partners know how to make use of the rich multimodal communication already happening, understanding when Mark is composing a message or he is assenting or dissenting with his blinks. Nonetheless having a physical and visually salient device can make Mark's communication intent much clearer, especially for conversation partners who do not know where to place their attentional focus. By augmenting embodied interaction through an external physical device, we are making it evident to other partners that an $\mathrm{AC}$ is conveying an action that has communicative intent. The sidekick's timer motion leverages a familiar cue that can support unfamiliar partners to understand that Mark is typing and he needs time to compose his message. Similar to other modalities such as screen-based emojis or LED lights [38], motion can grab immediate attention but it can also convey precise messages in an ambient, peripheral, and spatial fashion to overcome display resolution limitations.

Our evaluation approach allowed us to test the sidekick in-thewild but with some data limitations as we could not control the types of conversations that occurred. While we have not yet conducted a rigorous comparison of sidekick use with familiar and unfamiliar partners, we learned that the sidekick had the potential to make it easier for Mark to communicate specific things to unfamiliar partners, such as "I am typing," "hold on a minute" (while the sidekick moved), and "I am ready to participate" (once the sidekick stopped moving). But we learned that unfamiliar partners did not incorporate the sidekick into conversations seamlessly; it was actually mid-level conversation partners who incorporated the sidekick the most. Due to the nature of the conversations being mostly online we learned that the sidekick might be better introduced to new partners in this context with a message to tell new partners what they should focus on. For example, Mark could say "the red item on my device indicates that I am typing." Thinking about how different partners in Mark's circle have different communication abilities poses the opportunity to explore future communication technologies that are specific to partner types. For example, systems using more complex nonverbal gestures can be used with close partners who could spend more time learning a new system or a new language, or even providing content input [9].

\subsection{Barriers to long-term use and AT development}

The use of assistive technology (AT) has been reported to be low even when people have access to AT [7, 31, 32]. The reason for this is usually connected to usability barriers and social acceptabilitysocial barriers that impact AT use [36,37]. We identified some barriers to using the sidekick device long-term related to having to remember to plug it in and making sure the camera was set up at a right angle, both related to the daily set up routine. For instance, Mark told T: "We are going to have to remember to plug it in," illustrating that having to add extra steps into the daily technology set-up is not trivial and can be a barrier to using a new system. AT should aim to be mostly integrated to existing technology use, but this can be challenging when current AT systems such as AAC devices are not open to developers to build on and integrate new features in. Other developers have encountered the same limitation, the lack of a complete AAC functionality stack into which new developments can be built [9]. During our development phase we spent a considerable amount of time learning more about Mark's AAC device's capabilities and about his workflow - how he preferred to use a separate computer for Zoom and keep his AAC device mainly for communication. There were a lot of "unknowns" regarding how compatible his AAC device was to other peripheral devices. We decided to go with the stand-alone, head switch operated sidekick to make progress and make something work but this tension illustrated the boundaries of individual co-design, making it harder to recommend how bespoke technologies like this one can reach a wider audience.

Another factor that can impact AT use is access to proper maintenance of a device or troubleshooting over time. Maintenance of bespoke technology designs must be an important factor to consider to ensure it is used. T brought up this important point: "If this turns out to be a really helpful thing, then where do we get the technical support to keep the prototype functioning?" To address this, we open-sourced our design for others and also connected Mark to local makerspaces and volunteers working on making bespoke open-source assistive technology. The HCI researchers will continue to provide support for this device but having a long-term 
plan in place can make sure that maintenance is possible beyond the HCI researchers is also crucial. Though this tension of impact and maintenance is unresolvable with this project, we found that empowering Mark by making the design open and by keeping clear documentation is a starting point to ensure that end-users know what to ask for when seeking technical support. Future work may look at how existing online communities developing Do-it-Yourself AT [20] can continue maintaining and expanding motion-based AAC solutions.

\subsection{Accessibility of the long-term co-design process}

Carrying out our co-design collaboration over an extended period of time was beneficial, allowing us to develop valuable relationships and reflect on the process through accessible iteration and prototyping as found in prior work $[8,11]$. To collaborate with Mark and his close communication partners $\mathrm{T}$ and $\mathrm{D}$, enabling multiple feedback channels via email, video, drawing, collaboration boards and video chats was key in helping us carry out the co-design process. For example, we were able to take up the specific tools such as the Padlet ideation board that Mark recommended. Often, co-design is engaged with the assumption that designers provide resources during in-person workshops. We realized after the fact that we engaged privileged skillsets to move co-design online. While we may have been able to provide institutional access to digital tools and we provided the physical components necessary to prototype sidekicks, we took for granted the technical skill required to join us on these platforms. While Mark and his family were tech savvy, we did find that our explicit conversations with Mark about which communication tools would work for him were still important for us to have effective design sessions; co-design concerned the process, not only the prototype. As we recognized open communication about tools and techniques seemed to be a positive starting point, there is a need to explore co-design of bespoke technology during remote collaboration that can leverage different tools. For example, we learned a lot by dropping off preliminary prototypes with Mark and having the spatial conversational brainstorming sessions, where over a video call we learned the feasibility of different possibilities in fitting onto his wheelchair and into his overall space.

We also learned that less was indeed more. In co-design and ideation more generally, designers diverge to generate many different possibilities. Early in our process we came up with many drawings and ideas of where the sidekick could be placed and how it could be accessed and controlled (voice control, facial gesture recognizer, multiple buttons, etc.). We wanted to think of anything as possible as designers but as $\mathrm{T}$ and Mark shared, they have tried a lot. Harrington et al. critique "blue sky" ideation with critical race theory, that the underserved, black communities they worked with know what types of structural changes might enable access and ideating things that won't come to fruition can widen gaps between what different co-designers perceive of as ideal. Instead, Harrington et al. and Bennett et al. recommend understanding stories and rich accounts to recognize the knowledge and labor co-designers have already expended [3, 16]. In Mark's case, he and his family have already done the early exploration of figuring out ways to make communication easier and finding the right access modes. They have actually spent a lot of time and worked very hard, getting creative about many possible points of Mark's body to use for access. They wanted the HCI researchers to know what isn't going to work right away, and that it is very important to listen. We recommend that co-design actively incorporates 'what doesn't work' into design sessions. Further, in-depth listening to understand co-designers' process of iteration and ultimately determining the options that were not feasible was useful in helping us to not replicate these mistakes; in other words, lists of what not to do are helpful, but engaging the iterative everyday design that got to that point gave texture to the bad ideas that kept us from developing similar past solutions and unusable possibilities such that we did not attempt to replicate them.

\subsection{Limitations and Future work}

One limitation of our design process was that the hardware design iteration was done mostly by the $\mathrm{HCI}$ researchers as changes required $3 \mathrm{D}$ printing and 3D modeling and the motions were programmed directly to the sidekick's microcontroller. Next steps should include making the sidekick more robust to allow customization on the go. A future sidekick platform that enables authoring gestures via remote control could facilitate motion customizations by the user to produce changes on the go and explore new combination of gestures and without the need of HCI researchers in the loop. This flexible customization platform could also be furthered developed to provide ways to visualize other sidekick forms. Second, our codesigners had access to a lot of resources and had worked together for a long time to augment Mark's communication. As such, they quickly integrated into the design team. Future research should concern activating co-design that may support co-designers with different resources and experiences with DIY. Finally, making the sidekick more integrated with the AAC device in a way that it could be controlled by it is a clear opportunity for improvement and future work.

\section{CONCLUSION}

We explored how motion could support augmentative and alternative communication by co-designing and evaluating a physically expressive sidekick object with and for Mark. Using bespoke sidekicks that move in physical space as a form of aided nonverbal AAC can provide augmented communicators with an additional expressive output that can support them in managing conversation dynamics. By working closely with Mark and his family we also learned about the possible barriers to integrating a new device in daily life and in sharing our lessons, we look forward to future work in improving tools that support developers in building for AAC.

\section{ACKNOWLEDGMENTS}

We thank our participants and co-designers for making this work possible. We also thank Simran Jobanputra, Elizabeth Lister and Jared Santa Maria for their support in early ideation stages of this project. 


\section{REFERENCES}

[1] Lucy Anderson-Bashan, Benny Megidish, Hadas Erel, Iddo Wald, Guy Hoffman, Oren Zuckerman, and Andrey Grishko. 2018. The Greeting Machine: An Abstract Robotic Object for Opening Encounters. RO-MAN 2018 - 27th IEEE International Symposium on Robot and Human Interactive Communication: 595-602. https: //doi.org/10.1109/ROMAN.2018.8525516

[2] ASHA. Augmentative and Alternative Communication. Retrieved April 9, 2021 from https://www.asha.org/njc/aac/

[3] Cynthia L Bennett, Burren Peil, and Daniela K Rosner. 2019. Biographical Prototypes: Reimagining Recognition and Disability in Design. In Proceedings of the 2019 on Designing Interactive Systems Conference, 35-47.

[4] David R Beukelman, Pat Mirenda, and others. 1998. Augmentative and alternative communication. Paul H. Brookes Baltimore.

[5] Sarah W Blackstone. 1999. Circles of communication partners. Augmentative Communication News 12: 1-2.

[6] Gautam Bose, Marisa Lu, Lucas Ochoa, and Dan Lockton. 2019. Emoto: From phone to emotive robotic AI sidekick. TEI 2019 - Proceedings of the 13th International Conference on Tangible, Embedded, and Embodied Interaction: 451-457. https://doi.org/10.1145/3294109.3301260

[7] Katherine Deibel. 2013. A convenient heuristic model for understanding assistive technology adoption. Proceedings of the 15th International ACM SIGACCESS Conference on Computers and Accessibility, ASSETS 2013. https://doi.org/10. $1145 / 2513383.2513427$

[8] Kirsten Ellis, Ross De Vent, Reuben Kirkham, and Patrick Olivier. 2020. Bespoke Reflections: Creating a One-Handed Braille Keyboard. ASSETS 2020 - 22nd International ACM SIGACCESS Conference on Computers and Accessibility. https://doi.org/10.1145/3373625.3417019

[9] Alexander Fiannaca, Ann Paradiso, Mira Shah, and Meredith Ringel Morris. 2017. AACrobat: Using mobile devices to lower communication barriers and provide autonomy with gaze-based AAC. In Proceedings of the 2017 ACM Conference on Computer Supported Cooperative Work and Social Computing, 683-695.

[10] Melanie Fried-Oken, Charity Rowland, Glory Baker, Mayling Dixon, Carolyn Mills, Darlene Schultz, and Barry Oken. 2009. The effect of voice output on AAC supported conversations of persons with Alzheimer's disease. ACM Transactions on Accessible Computing 1, 3: 1-11. https://doi.org/10.1145/1497302.1497305

[11] Julia Galliers, Stephanie Wilson, Abi Roper, Naomi Cocks, Jane Marshall, Sam Muscroft, and Tim Pring. 2012. Words are not enough: Empowering people with aphasia in the design process. ACM International Conference Proceeding Series 1: 51-60. https://doi.org/10.1145/2347635.2347643

[12] Charles Goodwin. 2004. A Competent Speaker Who Can 't Speak: Journal of Linguistic Anthropology 14, 2: 151-170. https://doi.org/10.1525/jlin.2004.14.2.151

[13] Charles Goodwin. 2011. Contextures of action. Embodied interaction: Language and body in the material world: $182-193$.

[14] Google. Project Euphonia.

[15] Google. Paper Signals. Retrieved April 9, 2021 from https://papersignals. withgoogle.com/

[16] Christina Harrington, Sheena Erete, and Anne Marie Piper. 2019. Deconstructing Community-Based Collaborative Design. Proceedings of the ACM on HumanComputer Interaction 3, CSCW: 1-25. https://doi.org/10.1145/3359318

[17] D J Higginbotham, K Fulcher, and J Seale. 2016. Time and timing in interactions involving individuals with ALS, their unimpaired partners and their speech generating devices. The silent partner: 199-229.

[18] D Jeffery Higginbotham and D P Wilkins. 1999. Slipping through the timestream Social issues of time and timing in augmented interactions. Constructing (in) competence: Disabling evaluations in clinical and social interaction 2: 49-82.

[19] Guy Hoffman, Oren Zuckerman, Gilad Hirschberger, Michal Luria, and Tal ShaniSherman. 2015. Design and evaluation of a peripheral robotic conversation companion. In 2015 10th ACM/IEEE International Conference on Human-Robot Interaction (HRI), 3-10.

[20] Amy Hurst and Jasmine Tobias. 2011. Empowering individuals with do-it-yourself assistive technology. ASSETS'11: Proceedings of the 13th International ACM SIGACCESS Conference on Computers and Accessibility: 11-18. https://doi.org/ $10.1145 / 2049536.2049541$

[21] Seray B. Ibrahim, Asimina Vasalou, and Michael Clarke. 2018. Design Opportunities for AAC and Children with Severe Speech and Physical Impairments. Proceedings of the $2018 \mathrm{CHI}$ Conference on Human Factors in Computing Systems - CHI '18: 1-13. https://doi.org/10.1145/3173574.3173801

[22] Malte F Jung, Nikolas Martelaro, and Pamela J Hinds. 2015. Using robots to moderate team conflict: the case of repairing violations. In Proceedings of the Tenth Annual ACM/IEEE International Conference on Human-Robot Interaction 229-236.

[23] Shaun K. Kane, Barbara Linam-Church, Kyle Althoff, and Denise McCall. 2012. What we talk about: Designing a context-aware communication tool for people with aphasia. ASSETS'12 - Proceedings of the 14th International ACM SIGAC CESS Conference on Computers and Accessibility: 49-56. https://doi.org/10.1145/
2384916.2384926

[24] Mark L Knapp, Judith A Hall, and Terrence G Horgan. 2013. Nonverbal communication in human interaction. Cengage Learning.

[25] Arlene W Kraat. 1985. Communication Interaction between Aided and Natural Speakers: A State of the Art Report. Retrieved from https://www.lib.uwo.ca/cgi-bin/ezpauthn.cgi?url=http://search.proquest. com/docview/63124784?accountid=15115\%0Ahttp://vr2pk9sx9w.search. serialssolutions.com?ctx_ver=Z39.88-2004\&ctx enc=info:ofi/enc:UTF$8 \&$ rfr_id=info:sid/ProQ\%3Aeric\&rft_val_fmt=info:ofi/fmt

[26] Google Creative Lab. Teachable Machine. Retrieved April 12, 2021 from https: //teachablemachine.withgoogle.com/

[27] Hagen Lehmann, Joan Saez-Pons, Dag Sverre Syrdal, and Kerstin Dautenhahn. 2015. In good company? perception of movement synchrony of a nonanthropomorphic robot. PloS one 10, 5 .

[28] Michal Luria, Guy Hoffman, Benny Megidish, Oren Zuckerman, and Sung Park. 2016. Designing Vyo, a robotic Smart Home assistant: Bridging the gap between device and social agent. In 2016 25th IEEE International Symposium on Robot and Human Interactive Communication (RO-MAN), 1019-1025.

[29] Joohee Park, Young Woo Park, and Tek Jin Nam. 2014. Wrigglo: Shape-changing peripheral for interpersonal mobile communication. Conference on Human Factors in Computing Systems - Proceedings: 599-602. https://doi.org/10.1145/ 2559206.2574783

[30] Owain Pedgley. 2007. Capturing and analysing own design activity. Design Studies 28, 5: 463-483.

[31] B Phillips and H Zhao. 1993. Predictors of assistive technology abandonment. Assistive technology: the official journal of RESNA 5, 1: 36-45. https://doi.org/10. 1080/10400435.1993.10132205

[32] Bodil Ravneberg. 2012. Usability and abandonment of assistive technology. Journal of Assistive Technologies 6, 4: 259-269. https://doi.org/10.1108/ 17549451211285753

[33] Ben Robins, Kerstin Dautenhahn, and Paul Dickerson. 2009. From isolation to communication: a case study evaluation of robot assisted play for children with autism with a minimally expressive humanoid robot. In 2009 Second International Conferences on Advances in Computer-Human Interactions, 205-211.

[34] Elizabeth B-N Sanders and Pieter Jan Stappers. 2008. Co-creation and the new landscapes of design. Co-design 4, 1: 5-18.

[35] Jennifer M. Seale, Ann M. Bisantz, and Jeff Higginbotham. 2020. Interaction symmetry: Assessing augmented speaker and oral speaker performances across four tasks. AAC: Augmentative and Alternative Communication 36, 2: 82-94. https://doi.org/10.1080/07434618.2020.1782987

[36] Kristen Shinohara and Jacob O Wobbrock. 2011. In the shadow of misperception: assistive technology use and social interactions. In Proceedings of the SIGCHI Conference on Human Factors in Computing Systems, 705-714.

[37] Kristen Shinohara, Jacob O Wobbrock, and Wanda Pratt. 2018. Incorporating Social Factors in Accessible Design. In Proceedings of the 20th International ACM SIGACCESS Conference on Computers and Accessibility (ASSETS '18), 149-160. https://doi.org/10.1145/3234695.3236346

[38] Kiley Sobel, Alexander Fiannaca, Jon Campbell, Harish Kulkarni, Ann Paradiso, Ed Cutrell, and Meredith Ringel Morris. 2017. Exploring the Design Space of AAC Awareness Displays. In Proceedings of the 2017 CHI Conference on Human Factors in Computing Systems, 2890-2903.

[39] Interaction Research Studio. 2020. Yo-Yo Machines. Retrieved April 9, 2021 from https://www.yoyomachines.io/

[40] Hamish Tennent, Solace Shen, and Malte Jung. 2019. Micbot: A Peripheral Robotic Object to Shape Conversational Dynamics and Team Performance. ACM/IEEE International Conference on Human-Robot Interaction 2019-March, January: 133-142. https://doi.org/10.1109/HRI.2019.8673013

[41] Tobii Dynavox. 2020. Tobii Dynavox Partner Window. Retrieved June 4, 2020 from https://www.tobiidynavox.com/en-US/products/i-series/

[42] Stephanie Valencia, Michal Luria, Amy Pavel, Jefrey P Bigham, and Henny Admoni. 2021. Co-designing Socially Assistive Sidekicks for Motion-based AAC.

[43] Stephanie Valencia, Amy Pavel, Jared Santa Maria, Seunga (Gloria) Yu, Jeff Bigham, and Henny Admoni. 2020. Conversational Agency in Augmentative and Alternative Communication. In Proceedings of the 2020 CHI Conference on Human Factors in Computing Systems.

[44] Jacqueline Kory Westlund, Jin Joo Lee, Luke Plummer, Fardad Faridi, Jesse Gray, Matt Berlin, Harald Quintus-Bosz, Robert Hartmann, Mike Hess, Stacy Dyer, Kristopher Dos Santos, Sigurdur Orn Adalgeirsson, Goren Gordon, Samuel Spaulding, Marayna Martinez, Madhurima Das, Maryam Archie, Sooyeon Jeong, and Cynthia Breazeal. 2016. Tega: A social robot. ACM/IEEE International Conference on Human-Robot Interaction 2016-April: 561. https://doi.org/10.1109/ HRI.2016.7451856

[45] John Zimmerman, Jodi Forlizzi, and Shelley Evenson. 2007. Research through design as a method for interaction design research in HCI. In Proceedings of the SIGCHI conference on Human factors in computing systems, 493-502. 